\title{
AN OPINION MINING MODEL BASED ON USER PREFERENCES
}

\author{
Manuela Angioni, Andrea Devola, Mario Locci and Franco Tuveri \\ Center for Research and Scientific Studies in Sardinia, Bld. 1, Piscina Manna, Pula, Cagliari, Italy
}

\begin{abstract}
This paper proposes an Opinion Mining model, parameterized according to the reviewer profile. The work aims to highlight and resolve some issues resulting from previous activities in evaluating the goodness of the results obtained by the analysis of the reviews. A user profiling system provides the set of parameters to associate with the aspects and allows, working with the Opinion Mining system, to configure itself according to the user preferences.
\end{abstract}

\section{KEYWORDS}

Opinion Mining, Opinion Summarization, NLP, Semantic Categorization

\section{INTRODUCTION}

In a previous work (Angioni et al., 2016), we have proposed an ensemble of aspect-based Opinion Mining algorithms, using a lexicon-based approach, with a Matrix Factorization to improve the prediction of results in a recommendation system. In this context, we realized that it is necessary to adopt a user profiling system, applied to the user-related data, in order to avoid the differences expressed by the evaluators and the reviewers in the evaluation of the reviews and in the rating expressed. It is thus more likely that evaluators and reviewers having similar profiles express similar ratings about the same reviews. The user profiling system provides the set of parameters to associate with the aspects and it allows the Opinion Mining system to configure itself according to the user preferences. The analysis of the reviews has highlighted that users base their criticism on few aspects. In addition, different users focus on particular aspects rather than others. In our opinion, when a user is interested in a specific aspect spends more sentences to describe it. It is thus possible to capture the interest of a user by weighting their interests through the aspects he is talking about. The paper describes an Opinion Mining model, parameterized according to the user profile.

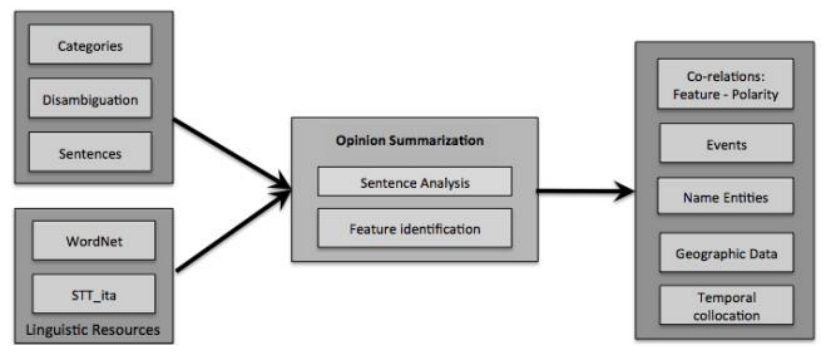

Figure 1. Opinion Summarization schema

The analysis of the reviews is illustrated in Figure 1, where are represented the linguistic resources, based on WordNet, used to perform the analysis and the text categorization, the semantic disambiguation and the analysis of sentences. In the Opinion Summarization phase the sentences are divided into subjective, usually expressing opinions, and objective. The identification of the features within the sentences allows associating the opinions to the aspects on which to focus the attention. A fundamental element is the features-polarity correlation, that allows evaluating the reviews. The Opinion Summarization phase is performed, through it, calculating the orientation, positive or negative, and referring it to the features or its attributes. 


\section{DATA ANALYSIS}

To test the functionality of the Opinion Mining system, a demonstrator has been developed that, using reviews related to a collection of films, is able to extract contextual information and features from textual resources. Some filters facilitate the consultation of reviews by offering the user only those of real interest or to generate and represent summaries in an effective and more immediately readable format.

The data used for the demo are the result of the integration of the information extracted from two different datasets: the "Movies and TV" dataset (He and McAuley, 2016) (McAuley et al., 2015), provided by Amazon in "json" format, and a subset of the IMDb database (Internet Movie Database, available at https://www.imdb.com/interfaces/) to supplement the data extracted from the Amazon DB.

A new database was thus built from the integration of the two original datasets, containing, for each film a limited number of reviews selected on the basis of their length and referred to 6 film genres: Thriller, Horror, Comedy, SciFi, Western and Drama. From the analysis of the reviews of the films, 6 main aspects have been identified: Picture, Sound, Script, Awards, Director, Actor/Actress, with a set of key terms, called features, mostly used in order to describe each aspect. The features are the terms of the reviews referred to the six aspects above mentioned and extracted with a procedure already described in (Angioni and Tuveri, 2011). The features, mapped on the aspects, are inserted in a database table.

\subsection{Semantic Analysis}

The semantic analysis of the movie reviews is carried out through a linguistic approach that involves the use and the implementation of different tools and proceeds through different phases, as shown in Figure 2.

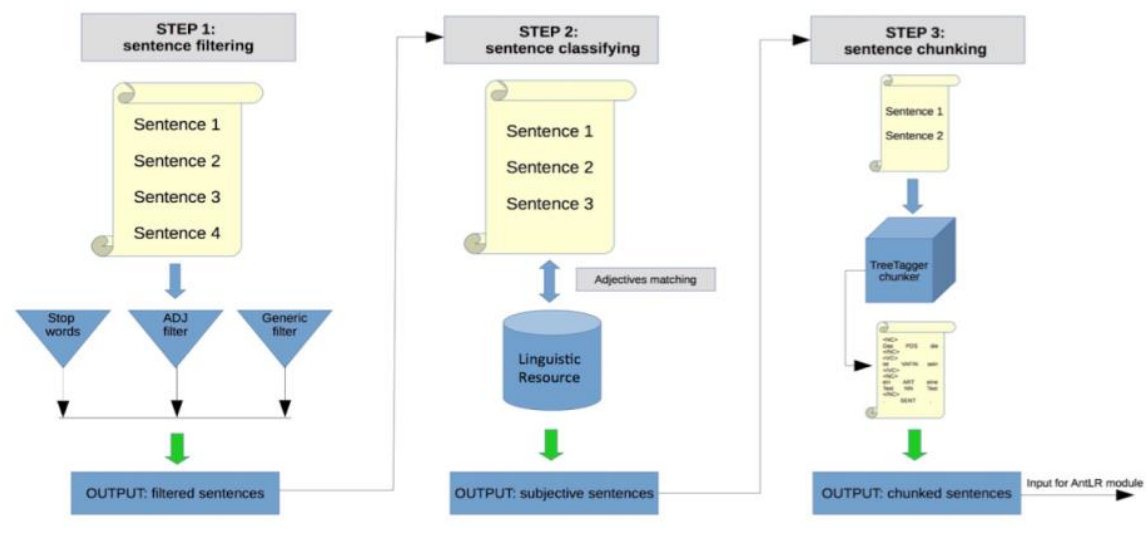

Figure 2. Text Analysis

\subsubsection{Sentence Filtering}

The sentence filtering is the first step of the analysis process of the reviews. It involves the identification of the sentences that compose a review. In this step the individual sentences are filtered, both through generic and specific filters, with the aim of searching for certain words or categories of words. As result, the portion of text to be analyzed is restricted due to the lower number of sentences to be analyzed.

The sentences are processed by several filters, some generic and some more specific, like the adjective_filter, named ADJ_filter, that allows the search for adjectives in each sentence received in input, or the stopwords_filter, for the identification of the stopwords. Purpose of those filters is to sift through the text of the sentence in search of certain words or categories of words, which will contribute to restrict the portion of text to be analyzed or the number of sentences to be considered. The ADJ_filter plays in this step a key role, as we'll see in the following step. The output, consisting of a small number of selected sentences, is used as input for the next phase of classification of the sentences. 


\subsubsection{Sentence Classifying}

The sentences, selected in the Step 1, are now classified in order to distinguish between "subjective sentences", which express a personal opinion, and "objective sentences". The classification takes place through the following steps:

- given a sentence, to which the "adjective filter" was previously applied, it is verified that this adjective is included in the available linguistic resource;

- if the match occurs, the sentence can be selected, and cataloged as a sentence with polarity;

- if the match does not occur, the sentence is discarded.

At the end of this phase, only the subjective sentences will be selected and pass to the last step.

\subsubsection{Sentence Chunking}

In the Step 3, the Chunk Analysis module analyzes the subjective sentences of the reviews. The Chunker is able to associate the terms, identified as features, with its attributes, that are the adjectives and the adverbs having subjective value in the sentence. In this phase, the TreeTagger Chunker (TTC) (Schmid, 1994) has been used. The text processed by the chunker is provided, in form of chunks, to ANTLR (Another Tool for Language Recognition, available at https://www.antlr.org/), a parser generator that performs the chunk analysis. For this purpose a grammar, described by a number of rules, has been defined. The rules allow analyzing the chunks identifying adjectives, adverbs, and verbs in a number of combinations by a given pattern. By providing the system with the ability to syntactically and semantically analyze sentences written in natural language, it is possible to support the collection of subjective opinions relating to the aspects and the features that characterize a text. Then the polarity value relative to each adjective and adverb connected to the features is calculated, through the polarity values expressed in SentiWordNet (Baccianella et al., 2010). For each review, the polarity values will be calculated for each of the aspects considered. The overall assessment is made through the computation of a score, based on the relevance of each aspect and calculated according to the terms associated with them. The result is weighted according to the user preferences: the user profile will influence in this way the film proposed by the application.

\section{CONCLUSION}

The work presented in this paper will carry on mainly through the integration of the text analysis module, composed by the Chunk Analysis and the Feature Evaluation, with the parameterization of results based on user profiles. We are working on the integration of the software modules that will lead to a test phase and to the evaluation of the results.

\section{REFERENCES}

Angioni, M., Tuveri, F., 2011. A Semantic Approach to the Extraction of Feature Terms. ICSOFT 2011, 6th International Conference on Software and Data Technologies.

Angioni, M., Clemente, M.L., Tuveri, F., 2016. Improving Predictions with an Ensemble of Linguistic Approach and Matrix Factorization. Web Information Systems and Technologies, Springer Valérie Monfort, Karl-Heinz Krempels, Tim A. Majc, vol. 246, Lecture Notes in Business Information Processing, pp. 169-190.

Baccianella, S., Esuli, A., Sebastiani, F., 2010. SentiWordNet 3.0: An Enhanced Lexical Resource for Sentiment Analysis and Opinion Mining. In Proceedings of LREC-10, 7th Conference on Language Resources and Evaluation. Valletta, MT, pp. 2200-2204.

R. He, J. McAuley, 2016. Ups and downs: Modeling the visual evolution of fashion trends with one-class collaborative filtering. In Proceedings of the $25^{\text {th }}$ International Conference on World Wide Web (WWW'16). pp. 507-517.

J. McAuley, C. Targett, J. Shi, A. van den Hengel, 2015. Image-based recommendations on styles and substitutes. SIGIR.

Schmid, H., 1994. Probabilistic Part-of-Speech Tagging Using Decision Trees. Proceedings of International Conference on New Methods in Language Processing. Manchester, UK.

Beck, K. and Ralph, J., 1994. Patterns Generates Architectures. Proceedings of European Conference of Object-Oriented Programming. Bologna, Italy, pp. 139-149. 